\title{
Low-Field Bi-Skyrmion Formation in a Noncentrosymmetric Chimney Ladder Ferromagnet
}

\author{
R. Takagi, ${ }^{1}$ X. Z. Yu, ${ }^{1}$ J. S. White, ${ }^{2}$ K. Shibata, ${ }^{1}$ Y. Kaneko, ${ }^{1}$ G. Tatara, ${ }^{1}$ H. M. Rønnow, ${ }^{3}$ Y. Tokura,,${ }^{1,4}$ and S. Seki ${ }^{1}$ \\ ${ }^{1}$ RIKEN Center for Emergent Matter Science(CEMS), Wako 351-0198, Japan \\ ${ }^{2}$ Laboratory for Neutron Scattering and Imaging (LNS), Paul Scherrer Institute (PSI), CH-5232 Villigen, Switzerland \\ ${ }^{3}$ Laboratory for Quantum Magnetism (LQM), Institute of Physics, École Polytechnique Fédérale de Lausanne (EPFL), \\ CH-1015 Lausanne, Switzerland \\ ${ }^{4}$ Department of Applied Physics, University of Tokyo, Bunkyo-ku 113-8656, Japan
}

(Received 1 September 2017; published 19 January 2018)

\begin{abstract}
The real-space spin texture and the relevant magnetic parameters were investigated for an easy-axis noncentrosymmetric ferromagnet $\mathrm{Cr}_{11} \mathrm{Ge}_{19}$ with Nowotny chimney ladder structure. Using Lorentz transmission electron microscopy, we report the formation of bi-Skyrmions, i.e., pairs of spin vortices with opposite magnetic helicities. The quantitative evaluation of the magnetocrystalline anisotropy and DzyaloshinskiiMoriya interaction (DMI) proves that the magnetic dipolar interaction plays a more important role than the DMI on the observed bi-Skyrmion formation. Notably, the critical magnetic field value required for the formation of bi-Skyrmions turned out to be extremely small in this system, which is ascribed to strong easyaxis anisotropy associated with the characteristic helix crystal structure. The family of Nowotny chimney ladder compounds may offer a unique material platform where two distinctive Skyrmion formation mechanisms favoring different topological spin textures can become simultaneously active.
\end{abstract}

DOI: 10.1103/PhysRevLett.120.037203

Magnetic Skyrmions are nanometric swirling spin textures with particle nature that have recently attracted much attention [1-8]. They are characterized by a topological index called the Skyrmion number [7], which is described by

$$
n_{\mathrm{sk}}=\frac{1}{4 \pi} \int \vec{n} \cdot\left(\frac{\partial \vec{n}}{\partial x} \times \frac{\partial \vec{n}}{\partial y}\right) d x d y,
$$

with $\vec{n}=\vec{m} /|\vec{m}|$ being the unit vector pointing along the local magnetization orientation $\vec{m}$. In metallic materials, electrons moving through Skyrmion spin texture gain a nontrivial quantum Berry phase, which generates a topological force on the underlying spin texture and enables unique current-induced Skyrmion manipulations [3,5,9-11]. Such features highlight Skyrmions as promising candidates for novel information technologies with high energy efficiency and information density.

To stabilize magnetic Skyrmions, several mechanisms have been proposed. One of the key sources is the Dzyaloshinskii-Moriya interaction (DMI) appearing in a noncentrosymmetric environment, which can stabilize various types of Skyrmion spin texture with $n_{\mathrm{sk}}=1$ depending on the underlying crystal lattice symmetry $[1,8]$. The early studies mostly focused on the vortex-type (i.e., Bloch-type) Skyrmions [Fig. 1(a)], emerging in the chiral cubic lattice system [2,4,12-14]. On the other hand, the appearance of different forms of Skyrmions has also been predicted for noncentrosymmetric uniaxial systems $[8,15,16]$, and the experimental discovery of Néel-type Skyrmions in $\mathrm{GaV}_{4} \mathrm{~S}_{8}$ with polar lattice symmetry [17], as well as anti-vortex-type
Skyrmions [Fig. 1(b)] in Heusler compounds with $D_{2 d}$ lattice symmetry [18], has recently been reported.

Another source for realizing the nontrivial Skyrmion spin texture is through a competition between the magnetic dipolar interaction (favoring the closed loop of local magnetization distribution to reduce stray field) and magnetocrystalline anisotropy [7,19-21]. For uniaxial ferromagnets with sufficiently strong easy-axis anisotropy, this competition can lead to the cylindrical ferromagnetic domain structure called magnetic bubble. Depending on the detail of the spin textures at the domain wall region, magnetic bubbles can often host nonzero Skyrmion numbers. For example, the experimental observation of biSkyrmions characterized by Skyrmion number $n_{\mathrm{sk}}=2$, i.e., a moleculelike pair of oppositely swirling vortex-type Skyrmions [Fig. 1(c)], has recently been reported for a few uniaxial centrosymmetric ferromagnets such as layered perovskite manganites [22] and $\mathrm{Mn}-\mathrm{Ni}-\mathrm{Ga}$ alloys [23].

Since such bi-Skyrmions or anti-vortex-type Skyrmions have rarely been observed experimentally, new material systems hosting such novel topological spin textures need to be further explored in the search for more useful properties and functions. Our target compound, $\mathrm{Cr}_{11} \mathrm{Ge}_{19}$ with metallic conductivity, belongs to a materials group called the Nowotny chimney ladder $A_{m} B_{n}$ (with $A$ and $B$ representing group 4-9 and group 13-15 elements, respectively) [24]. This group of binary intermetallic compounds commonly crystallize with a noncentrosymmetric tetragonal space group $P \overline{4} n 2$ (i.e., point group $D_{2 d}$ ), where $B$ 
(a)

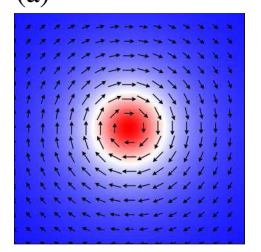

(b)

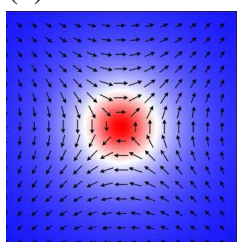

(c)

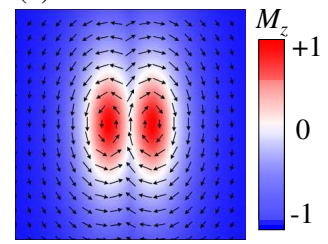

(d)

[001]

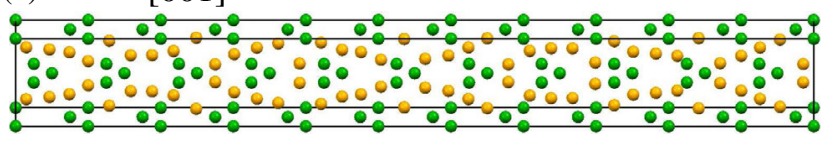

(e)

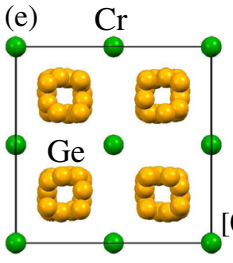

(f) $6 \longdiv { 1 0 \mathrm { K } }$
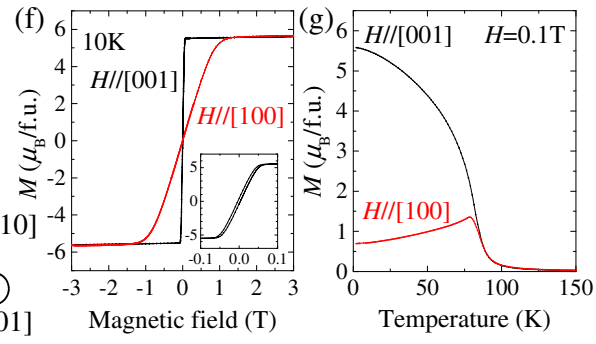

FIG. 1. Structural and magnetic properties of $\mathrm{Cr}_{11} \mathrm{Ge}_{19}$. (a)-(c) Schematic illustrations of various skyrmion spin textures, which represent (a) vortex-type Skyrmion, (b) anti-vortex-type Skyrmion, and (c) bi-Skyrmion, respectively. The background color indicates the out-of-plane component of local magnetization $M_{z}$. (d) Nowotny chimney ladder structure, characterized by a noncentrosymmetric tetragonal lattice with long [001] axis. (e) Arrangement of $\mathrm{Cr}$ and $\mathrm{Ge}$ atoms viewed along the [001] axis. The $\mathrm{Cr}$ and $\mathrm{Ge}$ atoms are shown as green and orange balls, respectively. (f) Magnetic field dependence of the magnetization measured under $H \|[100]$ (red) and $H \|[001]$ (black) at $10 \mathrm{~K}$. The inset shows the lower field data of the magnetization measured under $H \|[001]$. (g) Temperature dependence of $M$ measured under $\mu_{0} H=0.1 \mathrm{~T}$ for $H \|[100]$ (red) and $H \|[001]$ (black).

(=Ge) atoms form separate helices inside the helices of $A$ $(=\mathrm{Cr})$ atoms along the [001] axis, and with various helix periods $(m, n)$ as shown in Figs. $1(\mathrm{~d})$ and $1(\mathrm{e}) . \mathrm{Cr}_{11} \mathrm{Ge}_{19}$ is characterized by a ferromagnetic exchange interaction with easy-axis anisotropy as well as by the noncentrosymmetric $D_{2 d}$ lattice $[25,26]$, and, therefore, both types of Skyrmion formation mechanisms as introduced above may potentially be active depending on the relative magnitude of each magnetic interaction.

In the present Letter, we have investigated the real-space topological spin texture and associated magnetic interactions in this easy-axis noncentrosymmetric ferromagnet $\mathrm{Cr}_{11} \mathrm{Ge}_{19}$. By performing Lorentz transmission electron microscopy (LTEM) experiments, we found the formation of the bi-Skyrmion lattice within the (001) plane. The magnetic resonance and propagating spin-wave spectroscopy (PSWS) experiments have enabled the quantitative evaluation of both the magnetocrystalline anisotropy and DMI, which led us to the conclusion that the magnetic dipolar interaction plays a more important role than the DMI on the Skyrmion formation in $\mathrm{Cr}_{11} \mathrm{Ge}_{19}$. Notably, the critical magnetic field value required for the bi-Skyrmion formation turns out to be extremely low, probably due to the strong uniaxial anisotropy associated with the unique helix crystal structure.

$\mathrm{Cr}_{11} \mathrm{Ge}_{19}$ hosts a ferromagnetic ordering below $T_{c} \sim 90 \mathrm{~K}$. Figures $1(\mathrm{f})$ and $1(\mathrm{~g})$ show the $M-H$ (magnetizationmagnetic field) and $M-T$ (temperature) profiles for $H \|[100]$ and [001]. The $M-H$ profile shows tiny remanent magnetization [inset of Fig. 1(f)], and easy axis turns out to be along the [001] axis. The saturation magnetization $M_{s}$ is $0.51 \mu_{B} / \mathrm{Cr}$ at $10 \mathrm{~K}$. These magnetic properties are in good agreement with previous reports [25-27].

Next, we evaluated the magnitude of the magnetocrystalline anisotropy in $\mathrm{Cr}_{11} \mathrm{Ge}_{19}$ by using a magnetic resonance technique (See Supplemental Material for the details of the operating principle [28]) with the device structure as shown in Figs. 2(a) and 2(b), to examine if the criteria to generate magnetic bubble structures is satisfied. Figure 2(c) shows the magnetic field dependence of microwave absorption spectra $\left|\Delta S_{12}\right|$ measured under $H \|[001]$ at $10 \mathrm{~K}$. The resonance frequency shows a nonzero gap value at $H=0$ and linearly increases with $H$. Such magnetic field dependence can be well explained by a general ferromagnetic resonance behavior as described in the following.

The magnetic Hamiltonian for a ferromagnet with $D_{2 d}$ lattice symmetry in the continuum approximation can be described as $\mathcal{H}=\int E d \vec{r}$ with energy density $E$ given $[8,15,16]$ by (a)

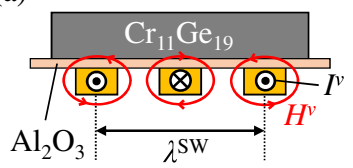

(c) $\left|\triangle S_{12}\right| \begin{array}{llll}1.2 & 0.8 & 0.4 & 0.0\end{array}$

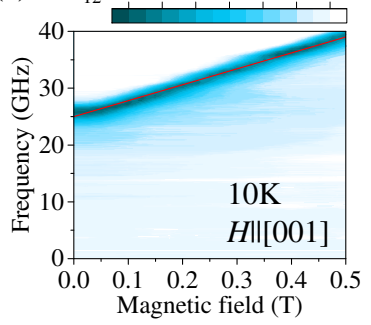

(b) $\quad 4 H$

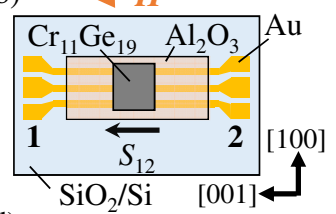

(d) 1.0

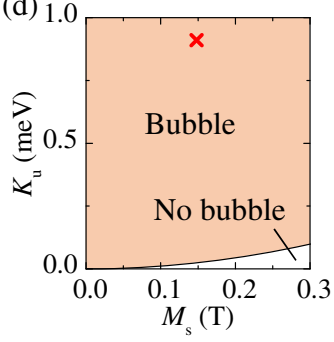

FIG. 2. (a) Side view and (b) top view of the device structure employed for magnetic resonance experiments. By injecting an oscillating electric current $I^{\nu}$ of gigahertz frequency [black arrows pointing along the out-of-plane direction in (a)] into the $\mathrm{Au}$ coplanar waveguide, an oscillating magnetic field $H^{\nu}$ is generated and a magnetic resonance is excited in $\mathrm{Cr}_{11} \mathrm{Ge}_{19}$. (c) Magnetic field dependence of the microwave absorption spectra at $10 \mathrm{~K}$ with $H \|[001]$. The background color represents the microwave absorption intensity $\left|\Delta S_{12}\right|$ associated with magnetic resonance. The red line is the result of theoretical fitting based on Eq. (3). (d) Condition required for magnetic bubble formation (i.e., $K_{u}>M_{s}^{2} / 2 \mu_{0}$ ), expressed in the $K_{u}$ (uniaxial magnetic anisotropy) $-M_{s}$ (saturated magnetization) plane. In the orange-shaded region, magnetic bubble structures can be stabilized. The cross mark represents the parameter obtained for $\mathrm{Cr}_{11} \mathrm{Ge}_{19}$. 


$$
E=\frac{J}{2}(\nabla \vec{S})^{2}-\tilde{D}-\frac{K}{2} S_{z}^{2}-\gamma \hbar \mu_{0} \vec{H} \cdot \vec{S},
$$

where $\tilde{D}=D\left[S_{z}\left(\partial S_{x} / \partial y\right)-S_{x}\left(\partial S_{z} / \partial y\right)+S_{z}\left(\partial S_{y} / \partial x\right)-\right.$ $S_{y}\left(\partial S_{z} / \partial x\right)$, and $J, D$, and $K$ describe the magnitudes of ferromagnetic exchange, DMI, and uniaxial anisotropy terms, respectively. $\vec{S}, \gamma, h=2 \pi \hbar$, and $\mu_{0}$ are the dimensionless vector spin density, the electron gyromagnetic ratio, Planck constant, and vacuum magnetic permeability, respectively. Considering a uniform magnetic excitation mode $(k \sim 0)$, the magnetic resonance frequency $\nu$ for $H \|[001]$ can be deduced as [30]

$$
\nu=K S+\gamma \hbar \mu_{0} H .
$$

The observed $H$ dependence of magnetic resonance frequency [Fig. 2(c)] can be well fitted on the basis of Eq. (3). By using the value of $S$ deduced from the saturated magnetization $M_{s}=\gamma \hbar S / V_{0}$ (with $V_{0}$ being the volume of formula unit cell) in the $M-H$ profile [Fig. 1(f)], we obtained $K_{u}=$ $K S^{2} / 2=0.91 \mathrm{meV}$ and $\gamma / 2 \pi=28 \mathrm{GHz} \mathrm{T}^{-1}$ from the fitting parameters. For the case of a plate-shaped ferromagnet with perpendicular magnetic anisotropy, magnetic bubble structures can be stabilized under $H \|[001]$ (i.e., along the out-of-plane easy-axis) when the uniaxial anisotropy energy $K_{u}$ exceeds the demagnetization field energy $M_{s}^{2} / 2 \mu_{0}$ [31]. As exhibited in Fig. 2(d), $K_{u}$ deduced for $\mathrm{Cr}_{11} \mathrm{Ge}_{19}$ is 1 order of magnitude larger than this critical value (i.e., $\left.K_{u} \gg M_{s}^{2} / 2 \mu_{0}\right)$, suggesting the possible formation of magnetic bubbles or Skyrmions in this compound.

On the basis of the above analysis, we performed a realspace imaging of the spin texture. Figures $3(a)-3(c)$ show the
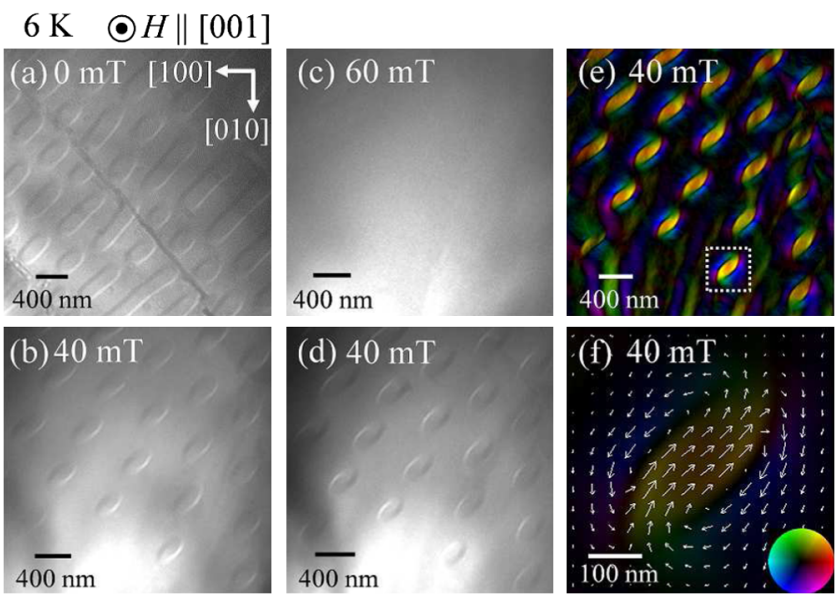

FIG. 3. Lorentz transmission electron microscopy (LTEM) images obtained for the (001) plane with a thickness of about $70 \mathrm{~nm}$ at $6 \mathrm{~K}$ with $H \|[001]$. (a)-(c) Overfocused LTEM images taken at (a) $\mu_{0} H=0$, (b) 40, and (c) $60 \mathrm{mT}$. (d) Underfocused LTEM image corresponding to (b). (e) Spatial distribution of the in-plane magnetization component, obtained by the TIE analysis [32] for (b) and (d). (f) Expanded view of a single bi-Skyrmion, corresponding to the boxed area of (e). The color wheel at the bottomright corner of (f) shows the direction (hue) and relative magnitude of the lateral magnetization. In (f), the local directions of in-plane magnetization component are also indicated by white arrows. Each field image was extracted from the same region of the sample. magnetic field dependence of the overfocused LTEM images in a thin plate form of $\mathrm{Cr}_{11} \mathrm{Ge}_{19}$ at $6 \mathrm{~K}$, which were obtained under an out-of-plane magnetic field along the [001] axis during a $H$-increasing run after zero field cooling. At zero magnetic field [Fig. 3(a)], the sample exhibited the mixed structure consisting of magnetic bubbles and magnetic stripe domains with a periodicity of $\lambda \sim 450 \mathrm{~nm}$ on average. With increasing magnetic field, the magnetic stripes break into fragments. At $40 \mathrm{mT}$, the magnetic stripes become completely replaced by the bubble lattice [Fig. 3(b)]. These magnetic bubbles disappear above $60 \mathrm{mT}$ [Fig. 3(c)], which indicates the formation of a uniform ferromagnetic domain. This magnetic field dependence is closely correlated to the $M-H$ profile shown in the inset of Fig. 1(f). To scrutinize the internal spin texture within the magnetic bubbles, we further performed the transport-of-intensity equation (TIE) analysis [32] on overfocused [Fig. 3(b)] and underfocused [Fig. 3(d)] LTEM images. Figures 3(e) and 3(f) indicate the obtained spatial distribution of the in-plane magnetization components, which reveals that the magnetic bubble is composed of a pair of spin vortices with opposite magnetic helicities (i.e., clockwise and counterclockwise manners of spin curls). By assuming the direction of the out-of-plane magnetization at the cores of each of the spin vortices, the obtained LTEM images evidence the formation of bi-Skyrmions as illustrated in Fig. 1(c). Here, each spin vortex becomes elliptic with its short axis (i.e., bonding direction connecting two paired Skyrmion cores) aligned along the same [110] orientation, and similar LTEM images have also been reported for other materials hosting bi-Skyrmions [22,23]. The observed alignment of the bi-Skyrmion orientation can reflect the in-plane magnetocrystalline anisotropy, while tiny additional symmetry breaking such as unintentional strain or slight tilting of $H$ [33] from the [001] axis (which was confirmed to be less than $1^{\circ}$ ) may also contribute here. The magnetic contrast of the bubbles in Fig. 3(b) exhibits pairs of bright and dark parts, which also agrees with the simulated pattern for a biSkyrmion as depicted in Fig. S2(f) in Supplemental Material [28]. Note that the detection of the out-of-plane spin component is not easy for the LTEM technique, and the further study by other complementary technique would be helpful for the definite experimental identification of the three-dimensional spin distribution.

To identify the Skyrmion formation mechanism in $\mathrm{Cr}_{11} \mathrm{Ge}_{19}$, we evaluated the magnitude of the DMI by investigating the propagation character of spin waves using a device structure as illustrated in Fig. 4(a). The operating principle of PSWS is described in Refs. [34-37] and the Supplemental Material [28]. Here, we adopted the measurement configuration with $H\left\|k^{\mathrm{sw}}\right\|[100]$ along the in-plane direction, with $k^{\mathrm{sw}}$ representing the wave vector of spin wave. In this case, the spin wave dispersion in the saturated ferromagnetic state is described [30,37-39] as

$$
\nu=\left[\operatorname{sgn}\left(\vec{k}^{\mathrm{sw}} \cdot \vec{H}\right)\right] \frac{2 D S k^{\mathrm{sw}}}{h}+\frac{C^{\mathrm{sym}}}{h},
$$

with $C^{\text {sym }}=J S\left(k^{\mathrm{sw}}\right)^{2}+\gamma H-K S$, which is schematically illustrated in Fig. 4(b). Since the first and second terms on 


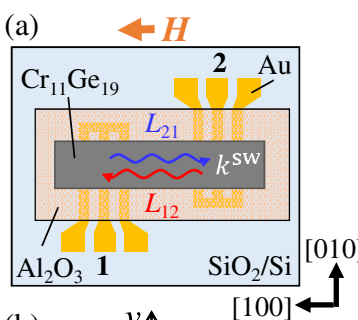

(b)
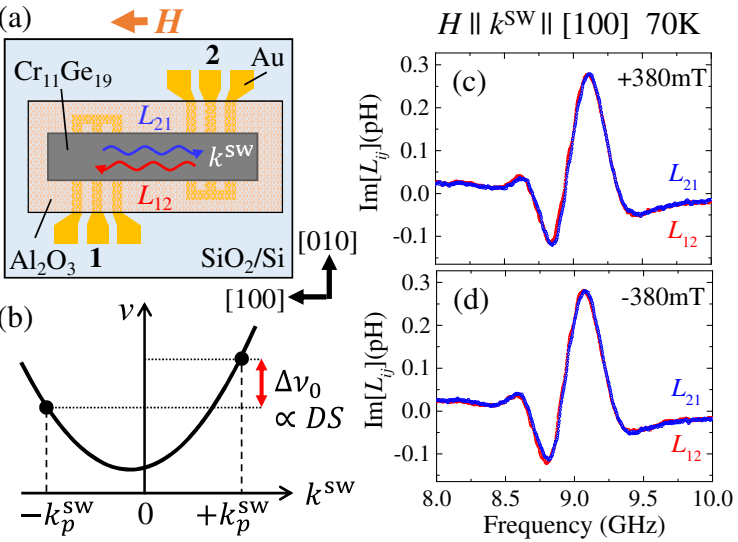

FIG. 4. (a) Schematic illustration of the device structure for propagating spin-wave spectroscopy (PSWS) experiments. Both the wave vector $k^{\mathrm{sw}}$ of propagating spin wave and the external magnetic field are set parallel to the in-plane [100] axis. Here, the spin wave with the wave number $k_{p}^{\mathrm{sw}}=2 \pi / \lambda^{\mathrm{sw}}$ is mainly excited, with $\lambda^{\text {sw }}$ being the periodicity of the waveguide pattern in Fig. 2(a). In the present measurements, we employed $\lambda^{\mathrm{sw}}=$ $12 \mu \mathrm{m}$ and $k_{p}^{\mathrm{sw}}=0.5 \mu \mathrm{m}^{-1}$. (b) Spin wave dispersion in the saturated ferromagnetic state with nonzero DMI, described by Eq. (4). (c),(d) Imaginary part of the mutual inductance $\Delta L_{21}$ and $\Delta L_{12}$ (representing spin waves propagating with wave vectors $+k^{\mathrm{sw}}$ and $-k^{\mathrm{sw}}$, respectively) measured at $70 \mathrm{~K}$ with (c) $\mu_{0} H=+380$ and (d) $\mu_{0} H=-380 \mathrm{mT}$.

the right side of Eq. (4) are odd and even functions of $k^{\mathrm{sw}}$, respectively, the former one proportional to $D$ makes the dispersion asymmetric and causes a nonzero frequency shift between oppositely propagating spin waves $\Delta \nu_{0}=$ $\nu\left(+\left|k^{\mathrm{sw}}\right|\right)-\nu\left(-\left|k^{\mathrm{sw}}\right|\right)$ described as

$$
\left|\Delta \nu_{0}\right|=\frac{4 D S\left|k^{\mathrm{sw}}\right|}{h} .
$$

Therefore, by measuring the frequency shift $\Delta \nu_{0}$ between spin waves propagating with the wave vectors $+k^{\mathrm{sw}}$ and $-k^{\mathrm{sw}}$, we can directly evaluate $D$ for $\mathrm{Cr}_{11} \mathrm{Ge}_{19}$ [Fig. 4(b)].

Figure 4(c) shows the imaginary part of the mutual inductance $\operatorname{Im}\left[\Delta L_{21}\right]$ and $\operatorname{Im}\left[\Delta L_{12}\right]$ measured at $70 \mathrm{~K}$ under $\mu_{0} H=+380 \mathrm{mT}$ (i.e., in the saturated ferromagnetic state [28]), which represent the spin waves propagating with the wave vectors $+k^{\mathrm{sw}}$ and $-k^{\mathrm{sw}}$, respectively. Both $\operatorname{Im}\left[\Delta L_{21}\right]$ and $\operatorname{Im}\left[\Delta L_{12}\right]$ show clear spin-wave signals around the magnetic resonance frequency, while a frequency shift $\Delta \nu_{0}$ between them was not discerned. According to Eq. (4), the sign of the DMI-induced $\Delta \nu_{0}$ should be reversed for the opposite direction of $H$, while such a behavior was not observed as shown in Fig. 4(d). Consequently, the frequency shift $\left|\Delta \nu_{0}\right|$ for $\mathrm{Cr}_{11} \mathrm{Ge}_{19}$ should be less than $10 \mathrm{MHz}$, which is within the error of the present measurement. From this result we deduce that the value of $D$ is at most $0.12 \mathrm{meV} \AA$ on the basis of Eq. (5).

In Table I, we list material parameters deduced for both $\mathrm{Cr}_{11} \mathrm{Ge}_{19}$, and other materials hosting various Skyrmion spin textures [18,22,23,39-41]. The value of $D$ for $\mathrm{Cr}_{11} \mathrm{Ge}_{19}$ is at least one order of magnitude smaller than those for chiral-lattice compounds with DMI-induced vortex-type Skyrmions, despite the larger magnitude of $J \sim T_{c} / M_{s}^{2}$. Moreover, for the $D_{2 d}$ lattice symmetry, the DMI should favor the anti-vortex-type Skyrmion [Fig. 1(b)], rather than the bi-Skyrmion spin texture $[8,18,42]$. These results suggest that the contribution of the DMI to the observed spin texture is of little significance in $\mathrm{Cr}_{11} \mathrm{Ge}_{19}$, and that the competition between the magnetic dipolar interaction and the magnetic anisotropy plays the crucial role for the bi-Skyrmion formation [43].

Previously, the experimental observation of bi-Skyrmions has been reported only for a few centrosymmetric ferromagnets such as layered perovskite manganites [22] and MnNi-Ga alloys [23]. Compared to these materials systems, $\mathrm{Cr}_{11} \mathrm{Ge}_{19}$ stands out due to several distinctive features. First, the critical magnetic field value required for the induction of bi-Skyrmion structure is extremely low. While bi-Skyrmions are formed only above $200 \mathrm{mT}$ in the previous compounds, in $\mathrm{Cr}_{11} \mathrm{Ge}_{19}$ bi-Skyrmions appear even under zero magnetic field [Fig. 3(a)] and the complete bi-Skyrmion lattice state is also achieved at $40 \mathrm{mT}$ [Fig. 3(b)]. This reflects the strong easy-axis magnetic anisotropy, as revealed by the magnetic resonance experiments [Fig. 2(d)], probably inherent to the unique helix crystal structure of Nowotny chimney ladder systems [Fig. 1(d)].

Second, $\mathrm{Cr}_{11} \mathrm{Ge}_{19}$ is the first noncentrosymmetric material to host bi-Skyrmion spin textures. While the contribution of the DMI is rather small for the present composition, the Nowotny chimney ladder phase allows a wide range of chemical tuning, with recent theoretical works suggesting that the magnitude of $D$ can be largely enhanced through the Fermi level modification across the band anticrossing points [44-46]. Since the DMI in the $D_{2 d}$

TABLE I. Magnetic parameters obtained for $\mathrm{Cr}_{11} \mathrm{Ge}_{19}$ in the present Letter and the ones previously reported for other materials hosting various Skyrmion spin textures [18,22,23,39-41]. The spin modulation period $\lambda$ for $\mathrm{Cr}_{11} \mathrm{Ge}_{19}$ was determined as an averaged period of the magnetic stripe structures shown in Fig. 3(a).

\begin{tabular}{|c|c|c|c|c|c|c|}
\hline & $D(\mathrm{meV} \AA)$ & $M_{s}(\mathrm{~T})$ & $T_{c}(\mathrm{~K})$ & $\lambda(\mathrm{nm})$ & Centro-symmetric? & Skyrmion type \\
\hline $\mathrm{La}_{2-2 x} \mathrm{Sr}_{1+2 x} \mathrm{Mn}_{2} \mathrm{O}_{7}$ & $\ldots$ & 0.16 & 110 & 180 & Yes & Bi-Skyrmion \\
\hline$\left(\mathrm{Mn}_{1-x} \mathrm{Ni}_{x}\right)_{65} \mathrm{Ga}_{35}$ & $\ldots$ & 0.023 & 350 & 180 & Yes & Bi-Skyrmion \\
\hline $\mathrm{Cr}_{11} \mathrm{Ge}_{19}$ & $<0.12$ & 0.073 & 90 & 450 & No $\left(D_{2 d}\right)$ & Bi-Skyrmion \\
\hline $\mathrm{Mn}_{1.4} \mathrm{Pt}_{0.9} \mathrm{Pd}_{0.1} \mathrm{Sn}$ & \multicolumn{2}{|c|}{ Unknown } & 400 & 135 & No $\left(D_{2 d}\right)$ & Anti-vortex \\
\hline $\mathrm{Cu}_{2} \mathrm{OSeO}_{3}$ & 1.9 & 0.13 & 59 & 62 & No (chiral) & Vortex \\
\hline $\mathrm{FeGe}$ & 4.6 & 0.23 & 278 & 70 & No (chiral) & Vortex \\
\hline
\end{tabular}


lattice system generally favors anti-vortex-type Skyrmions, there should be a critical $D$ value for the transition between the bi-Skyrmion and anti-vortex-type Skyrmion spin textures at which the dominant mechanism for the Skyrmion stabilization switches from the dipolar interaction to the DMI. The family of Nowotny chimney ladders can be a unique platform for such a topological transition of Skyrmions, and further exploration for their electromagnetic dynamics would be an interesting issue.

In summary, we experimentally revealed the emergence of the bi-Skyrmions in the easy-axis noncentrosymmetric ferromagnet $\mathrm{Cr}_{11} \mathrm{Ge}_{19}$ by means of real-space LTEM imaging. The quantitative evaluation of magnetocrystalline anisotropy and the DM interaction by magnetic resonance and PSWS experiments proved that the magnetic dipolar interaction plays a more critical role than the DM interaction on the present bi-Skyrmion formation. In $\mathrm{Cr}_{11} \mathrm{Ge}_{19}$, biSkyrmions can be generated even under zero magnetic field, which is an advantageous feature for their potential application and probably reflects the strong easy-axis anisotropy associated with the characteristic helix crystal structure. Considering their noncentrosymmetric $D_{2 d}$ lattice symmetry, the family of Nowotny chimney ladder compounds may offer a unique playground to investigate the competition between bi-Skyrmion and antiskyrmion spin textures with different topological charges and helicity structures arising from two distinctive skyrmion formation mechanisms.

The authors thank Y. Otani, K. Kondou, Y. Okamura, A. Kikkawa, and T. Arima for enlightening discussions and experimental support. This work was partly supported by the Mitsubishi Foundation, by JSPS Grants-In-Aid for Scientific Research (Grants No. 15H05458, No. 16K13842, and No. 17H05186), by the Swiss National Science Foundation (SNF) Sinergia network "NanoSkyrmionics," the SNF projects (Grants No. 153451 and No. 166298), and by the European Research Council project CONQUEST.

[1] U. K. Rößler, A. N. Bogdanov, and C. Pfleiderer, Nature (London) 442, 797 (2006).

[2] S. Mühlbauer, B. Binz, F. Jonietz, C. Pfleiderer, A. Rosch, A. Neubauer, R. Georgii, and P. Böni, Science 323, 915 (2009).

[3] F. Jonietz, S. Mühlbauer, C. Pfleiderer, A. Neubauer, W. Münzer, A. Bauer, T. Adams, R. Georgii, P. Böni, R. A. Duine, K. Everschor, M. Garst, and A. Rosch, Science 330, 1648 (2010).

[4] X. Z. Yu, Y. Onose, N. Kanazawa, J. H. Park, J. H. Han, Y. Matsui, N. Nagaosa, and Y. Tokura, Nature (London) 465, 901 (2010).

[5] A. Fert, V. Cros, and J. Sampaio, Nat. Nanotechnol. 8, 152 (2013).

[6] J. Sampaio, V. Cros, S. Rohart, A. Thiaville, and A. Fert, Nat. Nanotechnol. 8, 839 (2013).

[7] N. Nagaosa and Y. Tokura, Nat. Nanotechnol. 8, 899 (2013).

[8] A. Bogdanov and A. Hubert, J. Magn. Magn. Mater. 138, 255 (1994).
[9] T. Schulz, R. Ritz, A. Bauer, M. Halder, M. Wagner, C. Franz, C. Pfleiderer, K. Everschor, M. Garst, and A. Rosch, Nat. Phys. 8, 301 (2012).

[10] X. Z. Yu, N. Kanazawa, W. Z. Zhang, T. Nagai, T. Hara, K. Kimoto, Y. Matsui, Y. Onose, and Y. Tokura, Nat. Commun. 3, 988 (2012).

[11] J. Iwasaki, M. Mochizuki, and N. Nagaosa, Nat. Nanotechnol. 8, 742 (2013).

[12] X. Z. Yu, N. Kanazawa, Y. Onose, K. Kimoto, W. Z. Zhang, S. Ishiwata, Y. Matsui, and Y. Tokura, Nat. Mater. 10, 106 (2011).

[13] S. Seki, X. Z. Yu, S. Ishiwata, and Y. Tokura, Science 336, 198 (2012).

[14] Y. Tokunaga, X.Z. Yu, J. S. White, H. M. Rønnow, D. Morikawa, Y. Taguchi, and Y. Tokura, Nat. Commun. 6, 7638 (2015).

[15] A. N. Bogdanov and D. A. Yablonskiŭ, Zh. Eksp. Teor. Fiz. 95, 182 (1989).

[16] A. N. Bogdanov, U. K. Rößler, M. Wolf, and K.-H. Müller, Phys. Rev. B 66, 214410 (2002).

[17] I. Kézsmárki, S. Bordács, P. Milde, E. Neuber, L. M. Eng, J. S. White, H. M. Rønnow, C. D. Dewhurst, M. Mochizuki, K. Yanai, H. Nakamura, D. Ehlers, V. Tsurkan, and A. Loidl, Nat. Mater. 14, 1116 (2015).

[18] A. K. Nayak, V. Kumar, P. Werner, E. Pippel, R. Sahoo, F. Damay, U. K. Rößler, C. Felser, and S. S. P. Parkin, Nature (London) 548, 561 (2017).

[19] Y. S. Lin, P. J. Grundy, and E. A. Giess, Appl. Phys. Lett. 23, 485 (1973).

[20] T. Garel and S. Doniach, Phys. Rev. B 26, 325 (1982).

[21] X. Z. Yu, M. Mostovoy, Y. Tokunaga, W. Zhang, K. Kimoto, Y. Matsui, Y. Kaneko, N. Nagaosa, and Y. Tokura, Proc. Natl. Acad. Sci. U.S.A. 109, 8856 (2012).

[22] X. Z. Yu, Y. Tokunaga, Y. Kaneko, W. Z. Zhang, K. Kimoto, Y. Matsui, Y. Taguchi, and Y. Tokura, Nat. Commun. 5, 3198 (2014).

[23] W. Wang, Y. Zhang, G. Xu, L. Peng, B. Ding, Y. Wang, Z. Hou, X. Zhang, X. Li, E. Liu, S. Wang, J. Cai, F. Wang, J. Li, F. Hu, G. Wu, B. Shen, and X.-X. Zhang, Adv. Mater. 28, 6887 (2016).

[24] T. Caillat, J.-P. Fleurial, and A. Borshchevsky, J. Alloys Compd. 252, 12 (1997).

[25] N. J. Ghimire, M. A. McGuire, D. S. Parker, B. C. Sales, J.-Q. Yan, V. Keppens, M. Koehler, R. M. Latture, and D. Mandrus, Phys. Rev. B 85, 224405 (2012).

[26] H. Han, L. Zhang, X. Zhu, H. Du, M. Ge, L. Ling, L. Pi, C. Zhang, and Y. Zhang, Sci. Rep. 6, 39338 (2016).

[27] N. Jiang, Y. Nii, R. Ishii, Z. Hiroi, and Y. Onose, Phys. Rev. B 96, 144435 (2017).

[28] See Supplemental Material at http://link.aps.org/ supplemental/10.1103/PhysRevLett.120.037203 for details of the experimental setup and data analysis, which includes Ref. [29].

[29] M. Beleggia, M. A. Schofield, Y. Zhu, M. Malac, Z. Liu, and M. Freeman, Appl. Phys. Lett. 83, 1435 (2003).

[30] D. D. Stancil, Theory of Magnetostatic Waves (Springer, New York, 1993).

[31] U. Gianola, D. Smith, A. Thiele, and L. V. Uitert, IEEE Trans. Magn. 5, 558 (1969).

[32] M. Uchida, Y. Onose, Y. Matsui, and Y. Tokura, Science 311, 359 (2006). 
[33] J. C. T Lee, J. J. Chess, S. A. Montoya, X. Shi, N. Tamura, S. K. Mishra, P. Fischer, B. J. McMorran, S. K. Sinha, E. E. Fullerton, S. D. Kevan, and S. Roy, Appl. Phys. Lett. 109, 022402 (2016).

[34] V. Vlaminck and M. Bailleul, Science 322, 410 (2008).

[35] V. Vlaminck and M. Bailleul, Phys. Rev. B 81, 014425 (2010).

[36] Y. Iguchi, S. Uemura, K. Ueno, and Y. Onose, Phys. Rev. B 92, 184419 (2015).

[37] S. Seki, Y. Okamura, K. Kondou, K. Shibata, M. Kubota, R. Takagi, F. Kagawa, M. Kawasaki, G. Tatara, Y. Otani, and Y. Tokura, Phys. Rev. B 93, 235131 (2016).

[38] M. Kataoka, J. Phys. Soc. Jpn. 56, 3635 (1987).

[39] R. Takagi, D. Morikawa, K. Karube, N. Kanazawa, K. Shibata, G. Tatara, Y. Tokunaga, T. Arima, Y. Taguchi, Y. Tokura, and S. Seki, Phys. Rev. B 95, 220406(R) (2017).
[40] M. Kubota, H. Fujioka, K. Hirota, K. Ohoyama, Y. Moritomo, H. Yoshizawa, and Y. Endoh, J. Phys. Soc. Jpn. 69, 1606 (2000).

[41] J. F. Mitchell, D. N. Argyriou, A. Berger, K. E. Gray, R. Osborn, and U. Welp, J. Phys. Chem. B 105, 10731 (2001).

[42] The DMI with $D_{2 d}$ symmetry does not modify the total free energy of biskyrmion spin texture.

[43] We have also performed additional small-angle neutron scattering experiment for bulk form of single crystalline $\mathrm{Cr}_{11} \mathrm{Ge}_{19}$, while incommensurate magnetic Bragg reflections cannot be detected within the (001) plane for the range of $0.002 \mathrm{~nm}^{-1}<q<0.06 \mathrm{~nm}^{-1}$ (i.e., $314 \mathrm{~nm}>\lambda>10.5 \mathrm{~nm}$ ).

[44] F. Freimuth, S. Blügel, and Y. Mokrousov, J. Phys. Condens. Matter 26, 104202 (2014).

[45] T. Koretsune, N. Nagaosa, and R. Arita, Sci. Rep. 5, 13302 (2015).

[46] T. Kikuchi, T. Koretsune, R. Arita, and G. Tatara, Phys. Rev. Lett. 116, 247201 (2016). 